\title{
DIOPHANTINE EQUATIONS WITH BALANCING-LIKE SEQUENCES ASSOCIATED TO BROCARD-RAMANUJAN-TYPE PROBLEM
}

\author{
Manasi Kumari Sahukar and Gopal Krishna Panda \\ National Institute of Technology Rourkela, India
}

\begin{abstract}
In this paper, we deal with the Brocard-Ramanujan-type equations $A_{n_{1}} A_{n_{2}} \cdots A_{n_{k}} \pm 1=A_{m}$ or $G_{m}$ or $G_{m}^{2}$ where $\left\{A_{n}\right\}_{n \geq 0}$ and $\left\{G_{m}\right\}_{m \geq 0}$ are either balancing-like sequences or associated balancing-like sequences.
\end{abstract}

\section{Introduction}

A positive integer $B$ is a balancing number $([1])$ if $1+2+\cdots+(B-1)=$ $(B+1)+\cdots+(B+R)$ holds for some positive integer $R$. If $B$ is a balancing number, then $8 B^{2}+1$ is a perfect square and its positive square root is known as a Lucas-balancing number $([18,27])$. The $n$-th balancing number is denoted by $B_{n}$ and the balancing numbers satisfy the binary recurrence $B_{n+1}=6 B_{n}-B_{n-1}$ with initial terms $B_{0}=0, B_{1}=1$. The $n$-th Lucasbalancing number is denoted by $C_{n}$ and the Lucas-balancing numbers satisfy the same binary recurrence as that of balancing numbers with different initial terms $C_{0}=1, C_{1}=3$.

For any fixed positive integer $A>2$, the sequence $\left\{x_{n}\right\}$, defined recursively as $x_{n+1}=A x_{n}-x_{n-1}$ with initial terms $x_{0}=0, x_{1}=1$, is known as a balancing-like sequence and for each $n, D x_{n}^{2}+1$, where $D=\frac{A^{2}-4}{4}$, is a perfect rational square and its positive square root is known as the $n$-th Lucasbalancing-like number. The Lucas-balancing-like sequence also satisfy a recurrence identical with that of balancing-like sequence, but with different initial values $([19,29])$. We call the sequence $\left\{y_{n}\right\}$ defined by $y_{n}=\sqrt{\left(A^{2}-4\right) x_{n}^{2}+4}$,

2010 Mathematics Subject Classification. 11D45, 11 B39.

Key words and phrases. Brocard-Ramanujan equation, Balancing-like and associated balancing-like sequence, Pell-like and associated Pell-like sequence. 
an associated balancing-like sequence. It is easy to see that the sequence $\left\{y_{n}\right\}$ satisfies a binary recurrence identical with the balancing-like sequence but with initial terms $y_{0}=2, y_{1}=A$. The Binet forms of the balancing-like and the associated balancing-like sequences are

$$
x_{n}=\frac{\alpha^{n}-\beta^{n}}{\alpha-\beta}, y_{n}=\alpha^{n}+\beta^{n}
$$

respectively, where $\alpha=\frac{A+\sqrt{A^{2}-4}}{2}$ and $\beta=\frac{A-\sqrt{A^{2}-4}}{2}$. The Pell-like sequence $\left\{p_{n}\right\}$ and the associated Pell-like sequence $\left\{q_{n}\right\}$ corresponding to a balancinglike sequence $\left\{x_{n}\right\}$ are defined by $p_{2 n}=2 x_{n}, p_{2 n+1}=x_{n+1}-x_{n}, q_{2 n}=$ $\frac{x_{n+1}-x_{n-1}}{2}, q_{2 n+1}=x_{n+1}+x_{n}([20])$. We call the sequence $s_{n}=\left\{2 q_{n}\right\}$, the Lucas-Pell-like sequence.

If $A$ and $B$ are fixed nonzero coprime integers, then the sequence $\left\{u_{n}\right\}_{n \geq 0}$ defined recursively by $u_{n+1}=A u_{n}+B u_{n-1}$ with initial terms $u_{0}=0, u_{1}=1$ is known as a Lucas sequence. The corresponding associated Lucas sequence $\left\{v_{n}\right\}_{n \geq 0}$ satisfies an identical recurrence relation with initial terms $v_{0}=2$ and $v_{1}=\bar{A}$. The Binet forms of these sequences are

$$
u_{n}=\frac{\gamma^{n}-\delta^{n}}{\gamma-\delta}, v_{n}=\gamma^{n}+\delta^{n}
$$

respectively, where $\gamma=\frac{A+\sqrt{A^{2}+4 B}}{2}$ and $\delta=\frac{A-\sqrt{A^{2}+4 B}}{2}$. If $B=-1$, then the sequences $\left\{u_{n}\right\}$ and $\left\{v_{n}\right\}$ coincide with the balancing-like sequence $\left\{x_{n}\right\}$ and the associated balancing-like sequence $\left\{y_{n}\right\}$ respectively.

The well-known Brocard-Ramanujan problem consists of finding all positive integer solutions of the Diophantine equation

$$
n !+1=m^{2} \text {. }
$$

This problem was posed by Brocard $([5,6])$ and independently by Ramanujan $([25,26])$, unknowing of Brocard's query. The only known solutions of this problem are $(n, m)=(4,5),(5,11),(7,71)$ and it is still an open question whether there exists any other solution of (1.2). Overholt ([17]) showed that the fulfilment of weaker version of the abc-conjecture implies the finiteness of number of solutions of (1.2). Berndt and Galway ([3]) did not find further solutions of (1.2) for $8 \leq n \leq 10^{9}$ and recently, Matson and Robert ([15]) improved the upper bound to $10^{12}$.

For $A \in \mathbb{Z}$, Dabrowski ([8]) generalized the Brocard-Ramanujan problem and proved the finiteness of the integer solutions of $n !+A=m^{2}$. Berend and Harmse ([2]) studied the generalized version $n !=P(x)$ of $(1.2)$, where $P(x)$ is a polynomial, and showed that the later equation has only finitely many solutions if $P(x)$ has an irreducible factor of relatively large degree. Dabrowski and Ulas ([9]) worked on an equation of the form $y^{2}=B U_{n}+A$, where $U_{n}=f(1) f(2) \cdots f(n)$ and $f$ is an increasing function from $\mathbb{N}$ to $\mathbb{N}$. 
By replacing the consecutive natural numbers $1,2, \ldots, n$ of $n$ ! with terms of a Lucas sequence $\left\{u_{n}\right\}$, Luca and Shorey ([13]) proved that the Diophantine equation

$$
u_{n} u_{n+1} \cdots u_{n+k-1}+t=y^{l},
$$

where $t$ is not a perfect power, has finitely many solutions. Subsequently, Marques ([14]) proved that the Fibonacci version of Brocard-Ramanujan equation

$$
F_{n} F_{n+1} \cdots F_{n+k-1}+1=F_{m}^{2},
$$

has no solution in positive integers $m, n$ and $k \geq 2$. Generalizing the above equation, Szalay ([31]) studied the Diophantine equation

$$
H_{n_{1}} H_{n_{2}} \cdots H_{n_{k}}+1=H_{m}^{2}
$$

where $\left\{H_{n}\right\}$ is either Fibonacci sequence or Lucas sequence or balancing sequence. With a slight modification of the above equation, Pongsriiam $([23,24])$ solved the equations of the form

$$
A_{n_{1}} A_{n_{2}} \cdots A_{n_{k}} \pm 1=A_{m}^{2} \text { or } G_{m}^{2}
$$

and

$$
A_{n_{1}} \cdots A_{n_{k}} \pm 1=A_{m} \text { or } G_{m}
$$

where $\left\{A_{n}\right\}_{n \geq 0}$ and $\left\{G_{m}\right\}_{m \geq 0}$ are either the Fibonacci or the Lucas sequence. As an extension of the works of Marques ([14]), Szalay ([31]), Pongsriiam ([23]) and Pink and Szikszai ([21]) solved the Brocard-Ramanuja-type equations

$$
u_{n_{1}} u_{n_{2}} \cdots u_{n_{k}}+1=u_{m}^{2}
$$

and

$$
v_{n_{1}} v_{n_{2}} \cdots v_{n_{k}}+1=v_{m}^{2}
$$

except a certain case, where $\left\{u_{n}\right\}_{n \geq 0}$ and $\left\{v_{n}\right\}_{n \geq 0}$ are the Lucas and associated Lucas sequences corresponding to $B= \pm 1$ respectively.

Motivated by the above works, we study the Brocard-Ramanuja-type equation

$$
A_{n_{1}} \cdots A_{n_{k}} \pm 1=A_{m} \text { or } G_{m} \text { or } G_{m}^{2},
$$

where $\left\{A_{n}\right\}_{n \geq 0}$ and $\left\{G_{m}\right\}_{m \geq 0}$ are either balancing-like or associated balancing-like sequences.

\section{Preliminaries}

Let $a$ and $b$ be complex numbers, $a+b=\sqrt{R}, a b=Q, R$ and $Q$ are coprime nonzero integers and $\frac{a}{b}$ is not a root of unity. The Lehmer sequence is defined as

$$
U_{n}= \begin{cases}\frac{a^{n}-b^{n}}{a-b} & \text { if } n \text { is odd } \\ \frac{a^{n}-b^{n}}{a^{2}-b^{2}} & \text { if } n \text { is even }\end{cases}
$$


and its associated Lehmer sequence is

$$
V_{n}= \begin{cases}\frac{a^{n}+b^{n}}{a+b} & \text { if } n \text { is odd, } \\ a^{n}+b^{n} & \text { if } n \text { is even. }\end{cases}
$$

Lehmer numbers are generalizations of Lucas numbers on the divisibility properties and was studied by Lehmer himself in 1930. One can observe that Lucas numbers are also Lehmer numbers up to possible multiplication by a factor $a+b$. In particular,

$$
u_{n}= \begin{cases}U_{n} & \text { if } n \text { is odd } \\ (a+b) U_{n} & \text { if } n \text { is even. }\end{cases}
$$

Using the positive integer solutions of the quadratic Diophantine equation

$$
a x^{2}-b y^{2}=1,
$$

([32]), Keskin and Siar $([10])$ proved that if $(u, v)$ is the fundamental solution of (2.3), then all positive integer solutions of the $(2.3)$ are $(x, y)=\left(u\left(x_{n+1}-\right.\right.$ $\left.\left.x_{n}\right), v\left(x_{n+1}+x_{n}\right)\right)$ where $\left\{x_{n}\right\}$ is a balancing-like sequence corresponding to $A=4 a u^{2}-2$. Thus, the positive solutions of $(A+2) x^{2}-(A-2) y^{2}=4$ are given by $\left(x_{n+1}-x_{n}, x_{n+1}+x_{n}\right), n \geq 0$. Furthermore, it is easy to see that

$$
\begin{aligned}
& p_{2 n+1}=x_{n+1}-x_{n}=\frac{w^{2 n+1}+z^{2 n+1}}{w+z}, \\
& s_{2 n+1}=2\left(x_{n+1}+x_{n}\right)=2 \cdot \frac{w^{2 n+1}-z^{2 n+1}}{w-z}, \\
& s_{2 n}=w^{2 n}+z^{2 n}, \frac{p_{2 n}}{2}=\frac{w^{2 n}-z^{2 n}}{w^{2}-z^{2}},
\end{aligned}
$$

where $w=\frac{\sqrt{A+2}+\sqrt{A-2}}{2}$ and $z=\frac{\sqrt{A+2}-\sqrt{A-2}}{2}$. Since $(w+z)^{2}$ and $w z$ belong to set of non-zero integers and are coprime, the Lehmer and the associated Lehmer numbers corresponding to $w$ and $z$ are

$$
\begin{aligned}
& U_{2 n}=\frac{p_{2 n}}{2}, U_{2 n+1}=\frac{s_{2 n+1}}{2}, \\
& V_{2 n}=s_{2 n}, V_{2 n+1}=p_{2 n+1} .
\end{aligned}
$$

A prime number $p$ is a primitive prime divisor of the Lucas number $u_{n}$ if $p$ divides $u_{n}$, but does not divide $(\gamma-\delta)^{2} u_{2} \cdots u_{n-1}$. In Lehmer sequence, $p$ is a primitive prime divisor of $U_{n}$ if $p \mid U_{n}$ and $p \nmid\left(a^{2}-b^{2}\right)^{2} U_{2} \cdots U_{n-1}$. Since $u_{2 n}=u_{n} v_{n}$ by Lemma 2.3(1), it follows that a primitive prime divisor of $u_{2 n}$ is also a primitive prime divisor of $v_{n}$.

The following two lemmas deal with the existence conditions for the primitive prime divisors in the Lucas and the Lehmer sequences. These lemmas will be required in the main results of this paper. 
Lemma 2.1 ([7]). Suppose $\gamma$ and $\delta$ are real numbers such that $\gamma+\delta$ and $\gamma \delta$ are nonzero coprime integers and $\gamma \delta^{-1}$ is not a root of unity. If $n \neq 1,2,6$, then $u_{n}$ has a primitive prime divisor except when $n=12, \gamma+\delta=1$ and $\gamma \delta=-1$.

LEMMA 2.2 ([33]). If $a$ and $b$ are real numbers and $n>18$, then $U_{n}$ has a primitive divisor.

To explore the solutions of some Brocard-Ramanuja-type equations, we need to use some properties of Lucas and associated Lucas sequences, which are given in the following lemma.

LEMMA 2.3. If $m$ and $n$ are natural numbers, then

1. $u_{2 n}=u_{n} v_{n}, U_{2 n}=U_{n} V_{n}$,

2. $v_{n}^{2}-\left(A^{2}-4 B\right) u_{n}^{2}=4(\alpha \beta)^{n}$,

3. $u_{m} \mid u_{n}$ if and only if $m \mid n$,

4. $v_{m} \mid v_{n}$ if and only if $m \mid n$ and $\frac{n}{m}$ is odd,

5. if $m=2^{c} m^{\prime}$ and $n=2^{d} n^{\prime}, m^{\prime}$ and $n^{\prime}$ are odd, then

$$
\left(u_{m}, v_{n}\right)= \begin{cases}v_{(m, n)} & \text { if } c>d \\ 1 \text { or } 2 & \text { if } c \leq d .\end{cases}
$$

For the proofs of the assertions (1)-(4), see [28] and for the proof of (5) see $[16]$.

To establish the main results of this paper, we also need certain factorization properties of balancing-like and associated balancing-like numbers. The following lemma is important in this regard.

Lemma 2.4. The balancing-like sequence $\left\{x_{n}\right\}_{n \geq 0}$ and associated balancing-like sequence $\left\{y_{n}\right\}_{n \geq 0}$ corresponding to any $A>2$ satisfy

1. $x_{n}^{2}-1=x_{n-1} x_{n+1}, y_{n}^{2}-1=\frac{x_{3 n}}{x_{n}}$,

2. $x_{n}+1=\left\{\begin{array}{l}x_{\frac{n+1}{2}} y_{\frac{n-1}{2}} \\ \frac{1}{2} p_{n-1} s_{n+1}\end{array} \quad\right.$ if $n$ is is oven,

3. $x_{n}-1=\left\{\begin{array}{l}x_{\frac{n-1}{2}} y_{\frac{n+1}{2}} \\ \frac{1}{2} p_{n+1} s_{n-1}\end{array} \quad\right.$ if $n$ is is oven,

4. $y_{n}+1= \begin{cases}\frac{x_{3 n}}{x_{n}} & \text { if } n \text { is even, } \\ \frac{s_{3 n}}{s_{n}} & \text { if } n \text { is odd, }\end{cases}$

5. $y_{n}-1= \begin{cases}\frac{y_{3 n}}{y_{n}} & \text { if } n \text { is even, } \\ \frac{p_{3 n}}{p_{n}} & \text { if } n \text { is odd. }\end{cases}$

The above assertions can be proved using (1.1), (2.4), (2.5) and (2.6).

The following Lemma, which is also important for the development of main results, provides conditions under which balancing-like numbers are expressible as products of associated balancing-like numbers. 
LEMMA 2.5. For $m \geq 5$, the $m$-th balancing-like number $x_{m}$ corresponding to some $A>2$ can be written as

$$
x_{m}=y_{n_{1}} y_{n_{2}} \cdots y_{n_{k}},
$$

where $y_{n_{i}}$ is $n_{i}$-th associated balancing-like number, $0 \leq n_{1} \leq n_{2} \leq \cdots \leq n_{k}$, only if $m=2^{l}, l \geq 3$ or $m=3 \cdot 2^{l}, l \geq 1$.

Proof. If $m \geq 5$ is odd, then in view of Lemma 2.1, there exists an odd primitive prime divisor $p$ of $x_{m}$. By virtue of Lemma 2.3(5), $p$ does not divide any associated balancing-like number and therefore, $x_{m}$ cannot be expressed as a product of associated balancing-like numbers.

Now let $m \geq 5$ be even. Then we can write $m$ as $m=m_{1} \cdot 2^{l}, l \geq 1, m_{1} \geq 1$ is odd. If $m_{1} \geq 5$, then by Lemmas 2.1 and 2.3(5), there exists a prime $p$ such that $p \mid x_{m_{1}}$, but $p$ does not divide any associated balancing-like number. Since $m_{1} \mid m$, by Lemma 2.3(3) $p \mid x_{m}$ and this implies that $p \mid y_{n_{i}}$ for some $1 \leq i \leq k$, which contradicts the fact that $p$ does not divide any associated balancing-like number. Hence, for $m_{1} \geq 5, x_{m}$ is not expressible as product of associated balancing-like numbers. Thus, $m_{1}=1$ or 3 and consequently $m=2^{l}, l \geq 3$ or $m=3 \cdot 2^{l}, l \geq 1$.

If $m=2^{l}$ and $l \geq 3$, then

$$
x_{2^{l}}=y_{2^{l-1}} y_{2^{l-2}} \cdots y_{2} x_{2}=y_{2^{l-1}} y_{2^{l-2}} \cdots y_{2} y_{1},
$$

and in this case, $x_{2^{l}}$ is product of associated balancing-like numbers. Furthermore, if $m=3 \cdot 2^{l}$ and $l \geq 1$, then

$$
x_{3 \cdot 2^{l}}=y_{3 \cdot 2^{l-1}} y_{3 \cdot 2^{l-2}} \cdots y_{6} y_{3} x_{3} .
$$

Since $\left(x_{3}, y_{n}\right)=1$ or 2 for all $n$ by Lemma 2.3(5), $x_{3}$ is expressible as product of associated balancing-like numbers only if $x_{3}=A^{2}-1=2^{r}$ for some $r \geq 3$ since $A>2$. Hence, (2.9) holds only when $m=2^{l}, l \geq 3$ or $m=3 \cdot 2^{l}, l \geq 1$.

\section{Main Results}

In this section, we study some Brocard-Ramanujan-type equations that involve balancing-like and associated balancing-like numbers. These results are variants of the works done in $[14,31,23,24,21]$ for other sequences. In the proof of our main results, we use factorizations of balancing-like and associated balancing-like numbers, some results from [7,33] on the existence of primitive prime divisors of Lucas and Lehmer numbers and Lemma 2.5.

Throughout this section, $\left\{x_{n}\right\}_{n \geq 0}$ and $\left\{y_{n}\right\}_{n \geq 0}$ denote the balancinglike and associated balancing-like sequences respectively, $m, n_{1}, n_{2}, \ldots, n_{k}$ are nonnegative integers such that $n_{1} \leq n_{2} \leq \cdots \leq n_{k}$ and $k$ is a natural number. 
Theorem 3.1. If $A \neq 3$, then the equation

$$
x_{n_{1}} x_{n_{2}} \cdots x_{n_{k}}+1=y_{m}^{2}
$$

holds only if $k=1, n_{1}=3$ and $m=1$. Moreover, if $A=3$, then (3.1) holds only if $k=1, n_{1}=3, m=1$ or $k=1, n_{1}=2, m=0$.

Proof. Using Lemma 2.4, (3.1) can be written as

$$
x_{n_{1}} \cdots x_{n_{k}} x_{m}=x_{3 m} .
$$

If $m>2$, then by Lemma 2.1, $x_{3 m}$ has a primitive prime divisor $p$ that does not divide $x_{k}$ for $k<3 m$ and hence, if $n_{k}<3 m$, then $p$ does not divide any term on the left hand side of (3.2). If $n_{k}>3 m$, then there exists a primitive prime divisor of $x_{n_{k}}$ that does not divide $x_{3 m}$. Therefore, $3 m=n_{k}$ and hence (3.2) reduces to

$$
x_{n_{1}} \cdots x_{n_{k-1}} x_{m}=1
$$

which is not possible since $m>2$. If $m=1, y_{m}^{2}-1=A^{2}-1=x_{3}$ and for $m=0, y_{m}^{2}-1=2^{2}-1=3$, which holds only if $A=3$. If $m=2$, then (3.1) reduces to

$$
x_{n_{1}} x_{n_{2}} \cdots x_{n_{k}}=y_{2}^{2}-1=A^{4}-4 A^{2}+3 .
$$

One can check that $A^{4}-4 A^{2}+3<x_{5}$ and hence, $n_{k}$ cannot exceed 4 . But the only $x_{i}, 2 \leq i \leq 4$ that divides $A^{4}-4 A^{2}+3$ is $x_{3}=A^{2}-1$ and $A^{4}-4 A^{2}+3=x_{3}\left(A^{2}-3\right)$. Furthermore, $\left(A^{2}-3\right)<x_{3}$ and is divisible by $x_{2}=A$ only if $A=3$ and in this case $A^{4}-4 A^{2}+3=2 x_{3} x_{2}$ and 2 is not a balancing-like number. Hence (3.3) cannot hold for any $k$.

\section{Theorem 3.2. The Diophantine equation}

$$
y_{n_{1}} \cdots y_{n_{k}}+1=x_{m}^{2}
$$

has no solution for $m>5$. If $m \leq 5$, then for each $A>2$, (3.4) has at most a finite number of solutions.

Proof. If $m=3$, then $x_{3}^{2}-1=x_{2} x_{4}=y_{1}^{2} y_{2}$, which is a solution of (3.4) corresponding to $k=3, n_{1}=n_{2}=1, n_{3}=2$. If $m \leq 5$, then it is easy to see that for each $A>2,(3.4)$ has at most $5\left(2^{5}-1\right)=155$ solutions. Now assume that $m>5$. Using Lemma 2.4, (3.4) can be written as

$$
y_{n_{1}} \cdots y_{n_{k}}=x_{m+1} x_{m-1} .
$$

Since $y_{n}=\frac{x_{2 n}}{x_{n}}$, applying Lemma 2.3 to (3.5), we get

$$
x_{2 n_{1}} \cdots x_{2 n_{k}}=x_{m+1} x_{m-1} x_{n_{1}} \cdots x_{n_{k}} .
$$

If $m+1>2 n_{k}$, then by Lemma 2.1, no primitive prime divisor of $x_{m+1}$ divides any term on the left hand side of (3.6). If $m+1<2 n_{k}$, then no primitive 
prime divisor of $x_{2 n_{k}}$ divides any term on the right hand side of (3.6). Hence, $2 n_{k}=m+1$ which reduces (3.6) to

$$
x_{2 n_{1}} \cdots x_{2 n_{k-1}}=x_{m-1} x_{n_{1}} \cdots x_{n_{k}} .
$$

Since $n_{k}=\frac{m+1}{2}<m-1$, using the above argument repeatedly, one can conclude that $m-1=2 n_{k-1}, n_{k}=2 n_{k-2}$ and $n_{k-1}=2 n_{k-3}$, which imply that $m+1=2 n_{k}=4 n_{k-2}$ and $m-1=2 n_{k-1}=4 n_{k-3}$. Therefore, $4 \mid m-1$ and $4 \mid m+1$, which leads to $4 \mid(m+1)-(m-1)=2$, which is a contradiction. Hence, (3.4) has no solution for $m>5$.

\section{Theorem 3.3. The Diophantine equation}

$$
x_{n_{1}} x_{n_{2}} \cdots x_{n_{k}}-1=x_{m}
$$

has no solution for $m>19$. If $0 \leq m \leq 19$, then for each $A>2$, (3.7) can have at most finitely many solutions.

Proof. If $m=0$, then $x_{0}+1=x_{1}$ and if $m=3$, then $x_{3}+1=A^{2}=x_{2}^{2}$ leading to the solutions of (3.7). For $0 \leq m \leq 19$, using simple combinatorial argument, it is easy to see that (3.7) cannot have more than $20\left(2^{18}-1\right)$ possible solutions. Now, let $m>19$. If $m$ is odd, then by virtue of Lemma $2.4,(3.7)$ can be written as

$$
x_{n_{1}} x_{n_{2}} \cdots x_{n_{k}}=x_{m}+1=x_{\frac{m+1}{2}} y_{\frac{m-1}{2}} .
$$

Lemma 2.3(1) makes (3.8) equivalent to

$$
x_{n_{1}} x_{n_{2}} \cdots x_{n_{k}} x_{\frac{m-1}{2}}=x_{\frac{m+1}{2}} x_{m-1} .
$$

By Lemma 2.1, $x_{n}$ has a primitive prime divisor for all $n>6$. If $m-1<n_{k}$, then there exists a prime divisor $p$ such that $p \mid x_{n_{k}}$, but $p$ does not divide $x_{\frac{m+1}{2}}$ and $x_{m-1}$. If $m-1>n_{k}$, there exists a prime $p$ that divides $x_{m-1}$, but does not divide any $x_{n_{i}}$ for $1 \leq i \leq k$. Hence, $m-1=n_{k}$ which reduces (3.9) to

$$
x_{n_{1}} x_{n_{2}} \cdots x_{n_{k-1}} x_{\frac{m-1}{2}}=x_{\frac{m+1}{2}} .
$$

Since $\frac{m+1}{2}>\frac{m-1}{2}$, using the similar argument as above we conclude that $\frac{m+1}{2}=n_{k-1}$ and therefore, (3.10) takes the form

$$
x_{n_{1}} x_{n_{2}} \cdots x_{n_{k-2}} x_{\frac{m-1}{2}}=1 .
$$

Using the above equation, we get

$$
1=x_{n_{1}} x_{n_{2}} \cdots x_{n_{k-2}} x_{\frac{m-1}{2}} \geq x_{\frac{m-1}{2}}>A,
$$

which contradict our assumption $A>2$. If $m$ is even, then using Lemma 2.4, (3.7) can be written as

$$
x_{n_{1}} x_{n_{2}} \cdots x_{n_{k}}=x_{m}-1=\frac{1}{2} p_{m+1} s_{m-1} .
$$


In view of (2.3) and (2.4), the above equation can be written in terms of Lehmer and associated Lehmer numbers as

$$
U_{2 n_{1}} U_{2 n_{2}} \cdots U_{2 n_{k}}=V_{m+1} U_{m-1}
$$

and in view of Lemma 2.3(1), the last equation is equivalent to

$$
U_{2 n_{1}} U_{2 n_{2}} \cdots U_{2 n_{k}} U_{m+1}=U_{2 m+2} U_{m-1} \text {. }
$$

Since, by Lemma 2.2, the Lehmer number $U_{n}$ has a primitive prime divisor for $n>18$, it follows that $2 m+2=2 n_{k}$, and using Lemma 2.2 once more, we get $m-1=2 n_{k-1}$. Now, we conclude from (3.11) that

$$
1=U_{2 n_{1}} U_{2 n_{2}} \cdots U_{2 n_{k-2}} U_{m+1} \geq U_{m+1}>U_{3}
$$

which is not possible and hence, (3.7) has no solution for $m>19$.

Theorem 3.4. The Diophantine equation

$$
x_{n_{1}} x_{n_{2}} \cdots x_{n_{k}}+1=x_{m}
$$

has no solution for $m>17$ and (3.12) may be solvable for $m \leq 17$ depending on the values of $A$.

Proof. If $m=1$, then $x_{1}-1=x_{0}$, which is a solution of (3.12). If $m \leq 17$, it is easy to check the possible solutions of (3.12) for different values of $A$. Now assume that $m>17$. If $m$ is odd, then using Lemma 2.4 to (3.12), we get

$$
x_{n_{1}} x_{n_{2}} \cdots x_{n_{k}}=x_{\frac{m-1}{2}} y_{\frac{m+1}{2}} .
$$

Using Lemma 2.3(1), the above equation can be written as

$$
x_{n_{1}} x_{n_{2}} \cdots x_{n_{k}} x_{\frac{m+1}{2}}=x_{\frac{m-1}{2}} x_{m+1} .
$$

In view of Theorem 2.1, $m+1=n_{k}$ and hence (3.13) reduces to

$$
x_{n_{1}} x_{n_{2}} \cdots x_{n_{k-1}} x_{\frac{m+1}{2}}=x_{\frac{m-1}{2}},
$$

which is not possible since the left hand side is greater than right hand side.

If $m$ is even, then application of Lemma 2.4, (3.12) results in

$$
x_{n_{1}} x_{n_{2}} \cdots x_{n_{k}}=\frac{1}{2} p_{m-1} s_{m+1} .
$$

Using (2.3) and (2.4), the above equation can be written in terms of Lehmer and associated Lehmer numbers as

$$
U_{2 n_{1}} U_{2 n_{2}} \cdots U_{2 n_{k}}=V_{m-1} U_{m+1} .
$$

An application of Lemma 2.3(1) makes the last equation equivalent to

$$
U_{2 n_{1}} U_{2 n_{2}} \cdots U_{2 n_{k}} U_{m-1}=U_{2 m-2} U_{m+1} .
$$


Applying Lemma 2.2 to (3.14), we get $2 m-2=2 n_{k}$. Using Lemma 2.2 once more, we get $m+1=2 n_{k-1}$, and this reduces (3.14) to

$$
U_{2 n_{1}} U_{2 n_{2}} \cdots U_{2 n_{k-2}} U_{m-1}=1
$$

which is not possible since $U_{m-1}>1$ for $m>17$. Hence, (3.12) has no solution for $m>17$.

\section{THEOREM 3.5. The Diophantine equation}

$$
y_{n_{1}} y_{n_{2}} \cdots y_{n_{k}}-1=y_{m}
$$

has no solution for $m>12$.

PRoOF. If $m>12$ is even, then using Lemma 2.4, (3.16) can be written as

$$
y_{n_{1}} y_{n_{2}} \cdots y_{n_{k}} x_{\frac{m}{2}}=x_{\frac{3 m}{2}} .
$$

In view of Lemma 2.3(1), (3.17) is equivalent to

$$
x_{2 n_{1}} x_{2 n_{2}} \cdots x_{2 n_{k}} x_{\frac{m}{2}}=x_{\frac{3 m}{2}} x_{n_{1}} x_{n_{2}} \cdots x_{n_{k}} .
$$

An application of Lemma 2.1 to (3.18) results in $\frac{3 m}{2}=2 n_{k}$ and (3.18) is now reduced to

$$
x_{2 n_{1}} x_{2 n_{2}} \cdots x_{2 n_{k-1}} x_{\frac{m}{2}}=x_{n_{1}} x_{n_{2}} \cdots x_{n_{k}} .
$$

Since $7 \leq \frac{m}{2}=\frac{2 n_{k}}{3}<n_{k}$, similar to the last case, one can use Lemma 2.1 to obtain $n_{k}=2 n_{k-1}$ and hence, $n_{k-1}=\frac{n_{k}}{2}$ and (3.19) further reduces to

$$
y_{n_{1}} y_{n_{2}} \cdots y_{n_{k-2}} x_{\frac{m}{2}}=x_{n_{k-1}} .
$$

But, $n_{k-1}=\frac{3 m}{8}<\frac{m}{2}$ implies that $y_{n_{1}} y_{n_{2}} \cdots y_{n_{k-2}} x_{\frac{m}{2}}<x_{\frac{m}{2}}$ which is absurd.

If $m>12$ is odd, then using Lemma 2.4, (3.16) can be written as

$$
y_{n_{1}} y_{n_{2}} \cdots y_{n_{k}}=\frac{p_{3 m}}{p_{m}} .
$$

Since $y_{n}=s_{2 n}$, the above equation leads to

$$
s_{2 n_{1}} s_{2 n_{2}} \cdots s_{2 n_{k}} p_{m}=p_{3 m}
$$

which, in terms of Lehmer and associated Lehmer numbers, can be written as

$$
V_{2 n_{1}} V_{2 n_{2}} \cdots V_{2 n_{k}} V_{m}=V_{3 m}
$$

An application of Lemma 2.2 to (3.20) gives $3 m=2 n_{k}$ and reduces (3.20) to

$$
V_{2 n_{1}} V_{2 n_{2}} \cdots V_{2 n_{k-1}} V_{m}=1
$$

which implies that $1 \geq V_{m}>V_{12}$ as $m>12$. But, this is not possible. Hence, (3.16) has no solution for $m>12$. 
Theorem 3.6. The Diophantine equation

$$
y_{n_{1}} y_{n_{2}} \cdots y_{n_{k}}+1=y_{m}
$$

has no solution for $m>18$.

Proof. If $m>18$ is even, then using Lemma 2.4, (3.21) can be written as

$$
y_{n_{1}} y_{n_{2}} \cdots y_{n_{k}} y_{\frac{m}{2}}=y_{\frac{3 m}{2}} .
$$

By the help of Lemma 2.1, (3.22) gives $\frac{3 m}{2}=n_{k}$ and thus, (3.22) reduces to

$$
y_{n_{1}} y_{n_{2}} \cdots y_{n_{k-1}} y \frac{m}{2}=1
$$

which gives $1 \geq y_{\frac{m}{2}}>y_{9}$. But, this is not possible.

If $m>18$ is odd, then using Lemma 2.4 in (3.21), we get

$$
y_{n_{1}} y_{n_{2}} \cdots y_{n_{k}}=\frac{s_{3 m}}{s_{m}} .
$$

Since $y_{n}=s_{2 n},(3.23)$ is equivalent to

$$
s_{2 n_{1}} s_{2 n_{2}} \cdots s_{2 n_{k}} s_{m}=s_{3 m}
$$

and converting in terms of Lehmer and associated Lehmer numbers, the last equation is equivalent to

$$
V_{2 n_{1}} V_{2 n_{2}} \cdots V_{2 n_{k}} U_{m}=U_{3 m} .
$$

Applying Lemma 2.3(1) in (3.24), we get

$$
V_{2 n_{1}} V_{2 n_{2}} \cdots V_{2 n_{k-2}} U_{4 n_{k-1}} U_{4 n_{k}} U_{m}=U_{3 m} U_{2 n_{k}} U_{2 n_{k-1}} .
$$

An application of Lemma 2.2 in (3.25) gives $3 m=4 n_{k}$ and hence, $2 n_{k}=$ $\frac{3 m}{2}>m>18$. Using Lemma 2.2 in (3.25) once more, we get $2 n_{k}=4 n_{k-1}$ and (3.25) reduces to

$$
V_{2 n_{1}} V_{2 n_{2}} \cdots V_{2 n_{k-2}} U_{m}=U_{2 n_{k-1}} .
$$

Since $2 n_{k-1}=n_{k}=\frac{3 m}{4}<m$, the left hand side of (3.26) is greater than right hand side, which contradicts (3.26). Hence, no solution of (3.21) exists for $m>18$.

Theorem 3.7. The Diophantine equation

$$
x_{n_{1}} x_{n_{2}} \cdots x_{n_{k}}+1=y_{m}
$$

has no solution for $m>6$ and it can have at most finite number of solutions for $m \leq 6$ for each $A$. 
PRoOF. If $m=0$, then $x_{1}+1=y_{0}$, which is a solution of (3.27) corresponding to $k=1$ and $n_{1}=1$. One can check that (3.27) has at most $6\left(2^{5}-1\right)=186$ solutions if $m \leq 6$ for each $A$. Now assume that $m>6$. If $m$ is even, then using Lemma 2.4, (3.27) can be written as

$$
x_{n_{1}} x_{n_{2}} \cdots x_{n_{k}} y_{\frac{m}{2}}=y_{\frac{3 m}{2}} .
$$

Lemma 2.3(1) makes (3.28) equivalent to

$$
x_{n_{1}} x_{n_{2}} \cdots x_{n_{k}} x_{m} x_{\frac{3 m}{2}}=x_{3 m} x_{\frac{m}{2}} .
$$

Lemma 2.1 applied to (3.29) gives $3 m=n_{k}$ and this reduces (3.29) to

$$
x_{n_{1}} x_{n_{2}} \cdots x_{n_{k-1}} x_{m} x_{\frac{3 m}{2}}=x_{\frac{m}{2}}
$$

which is not possible since the left hand side is greater than the right side.

If $m$ is odd, then with the help of Lemma $2.4,(3.27)$ can be written as

$$
x_{n_{1}} x_{n_{2}} \cdots x_{n_{k}}=\frac{s_{3 m}}{s_{m}}
$$

which, in terms of Lehmer and associated Lehmer numbers, is equivalent to

$$
U_{n_{1}} U_{n_{2}} \cdots U_{n_{k}} U_{m}=U_{3 m} .
$$

Using Lemma 2.2 in (3.31), we obtain $n_{k}=3 m$ and (3.31) reduces to

$$
U_{n_{1}} U_{n_{2}} \cdots U_{n_{k-1}} U_{m}=1
$$

which is not true since $U_{m}>1$ because of our assumption $m>6$. Hence, (3.27) has no solution for $m>6$.

THEOREM 3.8. The Diophantine equation

$$
x_{n_{1}} x_{n_{2}} \cdots x_{n_{k}}-1=y_{m}
$$

has no solution for $m>4$ and for each $A$, it has at most finite number of solutions if $m \leq 4$.

Proof. If $m=2$, then $x_{3}-1=y_{2}$ leading to a solution of (3.32) corresponding to $k=1, n_{1}=3$ and for $m \leq 4$, it is easy to see the finiteness of solutions for each $A$. Now assume that $m>4$. If $m$ is even, then using Lemma $2.4,(3.32)$ can be written as

$$
x_{n_{1}} x_{n_{2}} \cdots x_{n_{k}} x_{m} x_{\frac{m}{2}}=x_{\frac{3 m}{2}} .
$$

Now applying Lemma 2.1 to $(3.33)$, we get that $\frac{3 m}{2}=n_{k}$ and this reduces (3.33) to

$$
x_{n_{1}} x_{n_{2}} \cdots x_{n_{k-1}} x_{m} x_{\frac{3 m}{2}}=1,
$$

which is not possible since $x_{\frac{3 m}{2}}>1$ for $m>4$.

If $m$ is odd, then using Lemma 2.4, we can write (3.32) as

$$
x_{n_{1}} x_{n_{2}} \cdots x_{n_{k}}=\frac{p_{3 m}}{p_{m}}
$$


and this equation can be written in terms of Lehmer and associated Lehmer numbers as

$$
U_{n_{1}} U_{n_{2}} \cdots U_{n_{k}} V_{m}=V_{3 m} .
$$

Since $V_{n}=U_{2 n} / U_{n}$ from Lemma 2.3(1), the above equation takes the form

$$
U_{n_{1}} U_{n_{2}} \cdots U_{n_{k}} U_{2 m} U_{3 m}=U_{m} U_{6 m} .
$$

Using Lemma 2.2 in (3.34), we get $n_{k}=6 \mathrm{~m}$ and (3.34) reduces to

$$
U_{n_{1}} U_{n_{2}} \cdots U_{n_{k-1}} U_{2 m} U_{3 m}=U_{m}
$$

which does not hold since the left hand side is greater than the right hand side. Hence, (3.32) has no solution for $m>4$.

Theorem 3.9. If $m>18$, then the Diophantine equation

$$
y_{n_{1}} y_{n_{2}} \cdots y_{n_{k}}-1=x_{m}
$$

is solvable only if $m=2^{l+1}-1, l \geq 4$ or $3 \cdot 2^{l+1}-1, l \geq 2$. If $m<18$, then for each $A,(3.35)$ has only finite number of solutions.

Proof. For $m=1, y_{0}-1=x_{1}$, which is a solution of (3.35) corresponding to $k=1, n_{1}=0$ and it is easy to check the finiteness of solutions of (3.35) when $m<18$. Now assume that $m>18$. If $m$ is odd, then using Lemma 2.4, (3.35) can be written as

$$
y_{n_{1}} y_{n_{2}} \cdots y_{n_{k}}=x_{\frac{m+1}{2}} y_{\frac{m-1}{2}}
$$

and with the help of Lemma 2.3, the above equation takes the form

$$
y_{n_{1}} y_{n_{2}} \cdots y_{n_{k-1}} x_{2 n_{k}} x_{\frac{m-1}{2}}=x_{\frac{m+1}{2}} x_{n_{k}} x_{m-1} \text {. }
$$

Applying Lemma 2.1 to (3.36), we get $n_{k}=\frac{m-1}{2}$ and (3.36) reduces to

$$
y_{n_{1}} y_{n_{2}} \cdots y_{n_{k-2}} y_{n_{k-1}}=x_{\frac{m+1}{2}} \text {. }
$$

But, in view of Lemma 2.5, (3.37) holds only if $m=2^{l+1}-1, l \geq 4$ or $3 \cdot 2^{l+1}-1, l \geq 2$.

If $m$ is even, then using Lemma 2.4, (3.35) can be written as

$$
y_{n_{1}} y_{n_{2}} \cdots y_{n_{k}}=\frac{1}{2} p_{n-1} s_{n+1} .
$$

An use of Lemma 2.3, transforms the above equation to

$$
x_{2 n_{1}} x_{2 n_{2}} \cdots x_{2 n_{k}}=p_{m-1} s_{m+1} x_{n_{1}} x_{n_{2}} \cdots x_{n_{k}}
$$

and (3.38) can be written in terms of Lehmer and associated Lehmer numbers as

$$
U_{4 n_{1}} U_{4 n_{2}} \cdots U_{4 n_{k}}=V_{m-1} U_{m+1} U_{2 n_{1}} U_{2 n_{2}} \cdots U_{2 n_{k}} .
$$

With the help of Lemma 2.3, (3.39) takes the form

$$
U_{4 n_{1}} U_{4 n_{2}} \cdots U_{4 n_{k}} U_{m-1}=U_{2 m-2} U_{m+1} U_{2 n_{1}} U_{2 n_{2}} \cdots U_{2 n_{k}} .
$$


By the use of Lemma 2.2 to (3.40) gives $2 m-2=4 n_{k}$ and reduces (3.40) to

$$
U_{4 n_{1}} U_{4 n_{2}} \cdots U_{4 n_{k-1}}=U_{m+1} U_{2 n_{1}} U_{2 n_{2}} \cdots U_{2 n_{k-1}}
$$

and Lemma 2.3 reduces the above equation to

$$
V_{2 n_{1}} V_{2 n_{2}} \cdots V_{2 n_{k-1}}=U_{m+1} .
$$

Since $m+1$ is odd, using Lemma 2.2 for $m>17$, we can ascertain the existence of a primitive prime divisor of $U_{m+1}$ that does not divide any associated Lehmer number corresponding to that Lehmer sequence $\left\{U_{n}\right\}$, a contradiction to $(3.41)$.

Theorem 3.10. If $m>8$, then the Diophantine equation

$$
y_{n_{1}} y_{n_{2}} \cdots y_{n_{k}}+1=x_{m}
$$

is solvable if $m=2^{l}+1, l \geq 3$ or $3 \cdot 2^{l}+1, l \geq 2$. If $m \leq 8$, then for each $A$, (3.42) has at most finitely many solutions.

Proof. If $m=3, y_{2}+1=x_{3}$, which corresponds to a solution of (3.42) with $k=1, n_{1}=2$. For each $A$, it is easy to see that (3.42) has only finitely many solutions when $m \leq 8$. Now let $m>8$. If $m$ is odd, then using Lemma $2.4,(3.42)$ can be written as

$$
y_{n_{1}} y_{n_{2}} \cdots y_{n_{k}}=x_{\frac{m-1}{2}} y_{\frac{m+1}{2}} .
$$

Lemma 2.3 makes the above equation equivalent to

$$
y_{n_{1}} y_{n_{2}} \cdots y_{n_{k-1}} x_{2 n_{k}} x_{\frac{m+1}{2}}=x_{\frac{m-1}{2}} x_{n_{k}} x_{m+1} \text {. }
$$

By virtue of Lemma 2.1, (3.43) holds only if $n_{k}=\frac{m+1}{2}$ and consequently, (3.43) reduces to

$$
y_{n_{1}} y_{n_{2}} \cdots y_{n_{k-1}}=x_{\frac{m-1}{2}} .
$$

Using Lemma 2.5, one can see that (3.44) hold if $m=2^{l}+1, l \geq 3$ or $3 \cdot 2^{l}+1, l \geq 2$.

If $m$ is even, applying Lemma 2.4 to (3.42), we get

$$
y_{n_{1}} y_{n_{2}} \cdots y_{n_{k}}=p_{m+1} s_{m-1}
$$

and using Lemma 2.3, the above equation can be written as

$$
x_{2 n_{1}} x_{2 n_{2}} \cdots x_{2 n_{k}}=p_{m+1} s_{m-1} x_{n_{1}} x_{n_{2}} \cdots x_{n_{k}} .
$$

Writing (3.45) in terms of the Lehmer and the associated Lehmer numbers, we get

$$
U_{4 n_{1}} U_{4 n_{2}} \cdots U_{4 n_{k}}=V_{m+1} U_{m-1} U_{2 n_{1}} U_{2 n_{2}} \cdots U_{2 n_{k}} .
$$

The relation $U_{2 n}=U_{n} V_{n}$ makes it possible to write (3.46) as

$$
U_{4 n_{1}} U_{4 n_{2}} \cdots U_{4 n_{k}} U_{m+1}=U_{2 m+2} U_{m-1} U_{2 n_{1}} U_{2 n_{2}} \cdots U_{2 n_{k}} .
$$


Applying Lemma 2.2 to (3.47), we get $2 n_{k}=m+1$ and now (3.47) takes the form

$$
U_{4 n_{1}} U_{4 n_{2}} \cdots U_{4 n_{k-1}}=U_{m-1} U_{2 n_{1}} U_{2 n_{2}} \cdots U_{2 n_{k-1}}
$$

and using Lemma 2.3, we can reduce the above equation to

$$
V_{2 n_{1}} V_{2 n_{2}} \cdots V_{2 n_{k-1}}=U_{m-1} \text {. }
$$

Since $m-1>18$ is odd, Lemma 2.2 guarantees the existence of a primitive prime divisor $p$ of $U_{m-1}$, which does not divide any associated Lehmer number, a contradiction to (3.48).

ACKNowledgements.

It is our pleasure to thank both the anonymous referees for their valuable suggestions and comments which results in the presentation and accuracy of the paper vastly.

\section{REFERENCES}

[1] A. Behera and G. K. Panda, On the square roots of triangular numbers, Fibonacci Quart. 37 (1999), 98-105.

[2] D. Berend and J. E. Harmse, On polynomial-factorial Diophantine equations, Trans. Amer. Math. Soc. 358 (2005), 1741-1779.

[3] B. C. Berndt and W. F. Galway, On the Brocard-Ramanujan Diophantine equation $n !+1=m^{2}$, Ramanujan J. 4 (2006), 41-42.

[4] Y. Bilu, G. Hanrot and P. M. Voutier, Existence of primitive divisors of Lucas and Lehmer numbers, J. Reine Angew. Math. 539 (2001), 75-122.

[5] H. Brocard, Question 166, Nouv. Corresp. Math. 2 (1876), 287.

[6] H. Brocard, Question 1532, Nouv. Ann. Math. 4 (1885), 391.

[7] R. D. Carmichael, On the numerical factors of arithmetic forms $\alpha^{n} \pm \beta^{n}$, Ann. of Math. (2) 15 (1913), 30-70.

[8] A. Dabrowski, On the diophantine equation $n !+A=y^{2}$, Nieuw Arch. Wiskd. 14 (1996), 321-324.

[9] A. Dabrowski and M. Ulas, Variations on the Brocard-Ramanujan equation, J. Number Theory 133 (2013) 1168-1185.

[10] R. Keskin and Z. Siar, Positive integer solutions of some Diophantine equations in terms of integer sequences, Afr. Mat. 30 (2019), 181-194.

[11] M. A. Khan and H. Kwong, Some binomial identities associated with the generalized natural number sequence, Fibonacci Quart. 49 (2011), 57-65.

[12] D. H. Lehmer, An extended theory of Lucas' functions, Ann. of Math. 31 (1930), 419-448.

[13] F. Luca and T. N. Shorey, Diophantine equations with products of consecutive terms in Lucas sequences II, Acta Arith. 114 (2008), 298-311.

[14] D. Marques, The Fibonacci version of the Brocard-Ramanujan Diophantine equation, Port. Math. 68 (2011), 185-189.

[15] R. D. Matson, Brocard's problem 4th solution search utilizing quadratic residues (2017), http://unsolvedproblems.org/S99.pdf,

[16] W. L. McDaniel, The g.c.d in Lucas sequences and Lehmer sequences, Fibonacci Quart. 29 (1991), 24-29.

[17] M. Overholt, The Diophantine equation $n !+1=m^{2}$, Bull. Lond. Math. Soc. 25 (1993), 104. 
[18] G. K. Panda, Some fascinating properties of balancing numbers, In Proc. of Eleventh Internat. Conference on Fibonacci Numbers and Their Applications, Cong. Numer. 194 (2009), 185-189.

[19] G. K. Panda and S. S. Rout, A class of recurrent sequences exhibiting some exciting properties of balancing numbers, Int. J. Math. Comput. Sci. 6 (2012).

[20] G. K. Panda and S. S. Pradhan, Associate sequences of balancing-like sequence, Math. Rep. (Bucur.), to appear.

[21] I. Pink and M. Szikszai, A Brocard-Ramanujan-type equation with Lucas and associated Lucas sequences, Glas. Mat. Ser. III 52(72) (2017), 11-21.

[22] P. Pongsriiam, Factorization of Fibonacci numbers into products of Lucas numbers and related results, JP J. Algebra Number Theory Appl. 38(4) (2016), 363-372.

[23] P. Pongsriiam, Fibonacci and Lucas numbers which are one away from their products, Fibonacci Quart. 55(1) (2017), 29-40.

[24] P. Pongsriiam, Fibonacci and Lucas Numbers Associated with Brocard-Ramanujan Equation, Commun. Korean Math. Soc. 32(3) (2017), 511-522.

[25] S. Ramanujan, Question 469, J. Indian Math. Soc. 5 (1913), 59

[26] S. Ramanujan, Collected Papers, Chelsea, New York, (1962).

[27] P. K. Ray, Balancing and cobalancing numbers, Ph.D. Thesis, National Institute of Technology, Rourkela, India, 2009.

[28] P. Ribenboim, The book of prime number records, Springer-Verlag, New York (1988).

[29] S. S. Rout, Some generalizations and properties of balancing numbers, Ph.D. Thesis, National Institute of Technology, Rourkela, India, 2015.

[30] C. L. Stewart, Primitive divisors of Lucas and Lehmer numbers, in: Transcendence theory: advances and applications, eds. A. Baker and D.W. Masser, Academic Press, New York, (1977), 79-92.

[31] L. Szalay, Diophantine equations with binary recurrences associated to the BrocardRamanujan problem, Port. Math. 69 (2012), 213-220.

[32] D. T. Walker, On the Diophantine equation $m X^{2}-n Y^{2}= \pm 1$, Amer. Math. Monthly 74 (1967), 504-513.

[33] M. Ward, The intrinsic divisors of Lehmer numbers, Ann. of Math. (2) 62 (1955), 230-236.

M. K. Sahukar

Department of Mathematics

National Institute of Technology Rourkela

Odisha

India

E-mail: manasi.sahukar@gmail.com

G. K. Panda

Department of Mathematics

National Institute of Technology Rourkela

Odisha

India

E-mail: gkpanda_nit@rediffmail.com

Received: 4.2.2019.

Revised: 29.7.2019. 